\title{
Dynamic Modulation of Water Permeability in the Lens Aquaporin-0
}

\author{
Stephen Reichow and Tamir Gonen
}

Janelia Farm Research Campus, Howard Hughes Medical Institute, Ashburn VA USA

Water is central for life as it is solvates ions, helps deliver nutrients into a cell and remove waste products and support metabolism. Water channels known as aquaporins are responsible for the high flux of water into and out of cells. The precise control of water permeability is vital for cellular homeostasis. Not surprisingly, aquaporins are highly regulated. Aquaporin-0 is dynamically regulated by at least 3 distinct mechanisms: $\mathrm{pH}$, calcium/calmodulin and phosphorylation. These regulatory mechanisms will be discussed structurally and put in context of lens physiology and cellular homeostasis.

Freshly purified AQP0-CaM complex was diluted to $0.02 \mathrm{mg} / \mathrm{ml}$ with buffer containing $25 \mathrm{mM}$ HEPES (pH - 7.4), $5 \mathrm{mM} \mathrm{CaCl}_{2}$ and 0.3\% DM. $2 \mathrm{ml}$ was applied to a 400 mesh carbon coated EM grid (Ted Pella), stained with uranyl formate, blotted on filter paper and dried by laminar air flow. Negatively stained particles were visualized on a $120 \mathrm{kV}$ TEM (FEI) and images were recorded at a nominal magnification of $52,000 \times$ at the specimen level on film (Kodak SO-163) as tilted-pair images $\left(\mathrm{a}=0^{\circ}\right.$ and $50^{\circ}$ ). Images were digitized using a Nikon Coolscan 9000 with a $6.9 \mathrm{~mm}$ step size, and binned three times to yield a final pixel size of $3.98 \AA$ per pixel. Thon rings in the power spectra were used to select only those micrographs free of drift or significant astigmatism. The contrast transfer function (CTF) parameters were determined for each micrograph using the program CTFTILT. 11,720 particles were selected and processed in SPIDER for generating multivariate reference free projection averages and initial three-dimensional (3D) reconstruction using random conical tilt methods. Final refinement of the 3D density map was performed using FREALIGN. The final 3D density map was reconstructed with C2 symmetry and filtered to $25 \AA$ resolution as suggested by Fourier shell correlation (FSC) analysis.

A pseudo-atomic model of the AQP0-CaM complex was built by initially docking available crystal structures into the AQP0-CaM EM density map as follows. The transmembrane domain of the bovine AQP0 crystal structure (PDB 2B6P, residues 9-222) was manually placed into the $65 \AA \times 65 \AA \times 40 \AA$ region of the EM map with the cytoplasmic face of the AQP0 tetramer facing the two vacant lobe domains of the EM map. This initial placement was rotationally and translationally optimized by computational minimization routines in Chimera. The structure of $\mathrm{Ca}^{2+} / \mathrm{CaM}$ bound to the plant glutamate decarboxylase peptides ( $p t \mathrm{GAD}-\mathrm{CaM}$, PDB 1NWD) was manually placed within the two vacant lobes of the EM map in a symmetric fashion with the CaM binding clefts facing the AQP0 tetramer. The helical axis of the two $p t \mathrm{GAD}$ subunits were oriented toward the cytoplasmic ends of the last transmembrane helix of neighboring AQP0 monomers. This placement was optimized to avoid steric clashes with AQP0. The two $p t \mathrm{GAD}$ a-helical subunits were used as templates to model the AQP0 C-terminal helices from each subunit (residues 227 - 241). The C-terminal AQP0 a-helical residues L234 and L237 were structurally aligned with the $p t \mathrm{GAD}$ hydrophobic anchoring residues Trp485 and Ile482, respectively. The cytoplasmic linkers connecting the AQP0 C-terminal helices to the last transmembrane helix of each subunit (residues 223 - 226) were manually built and minimized using COOT. The CaM molecules and the AQP0 C-terminal linker and CaM binding domains (residues 223 241) were subjected to steepest decent minimization followed by conjugate gradient minimization routines in Chimera to regularize the geometries and remove steric interactions. The final model gave a cross-correlation of 0.93 compared to the experimental map. 
ITC experiments were performed on a MicroCal VP-ITC (GE Lifescience). AQP0 ${ }^{\mathrm{CBD}}$ peptides corresponding to the wild-type bovine sequence (residues $223-242$ ), the point mutants L234A and L237A, and the double mutant L234A/L237A were synthesized and purified to $>98 \%$ purity (Biomatik). The AQP0CBD peptides and CaM were dialyzed against 20mM HEPES (pH 7.0) and 5mM $\mathrm{CaCl} 2$ over 72 hours using a 1,000 molecular weight cutoff (MWCO) membrane (Spectra/Por). AQP0CBD peptide concentrations were adjusted to $500 \mathrm{mM}$ and titrated into the MicroCal cell containing $\mathrm{Ca} 2+/ \mathrm{CaM}$ at $20 \mathrm{mM}$ or dialysis buffer only held at $25^{\circ} \mathrm{C}$. ITC data was processed in Origin (OriginLab, Northampton MA) by subtracting the background heats of peptide mixing to buffer alone from heats obtained from mixing with $\mathrm{Ca}^{2+} / \mathrm{CaM}$ and thermodynamic parameters were obtained by fitting the data to a two-state binding isotherm.

\section{References:}

[1] Gonen T., Cheng Y., Sliz P., Hiroaki Y., Fujiyoshi Y., Harrison SC., Walz T (2005) Lipid-protein interactions in double layered two dimensional crystals of AQP0. Nature 438: 633 - 638.

[2] Reichow L.S. and Gonen T. (2008) Non-canonical binding of calmodulin to aquaporin-0: implications for channel regulation. Structure. 16: $1389-1398$.

[3] Gold MG, Reichow SL., O’Neill SE, Weisbrod CR, Langeberg LK, Bruce JE, Gonen T and Scott JD. (2012) AKAP2 anchors PKA with aquaporin-0 to support ocular lens transparency. EMBO Molecular Medicine 4: 15-26.

[4] The authors acknowledge funding from the Howard Hughes Medical Institute to T.G.

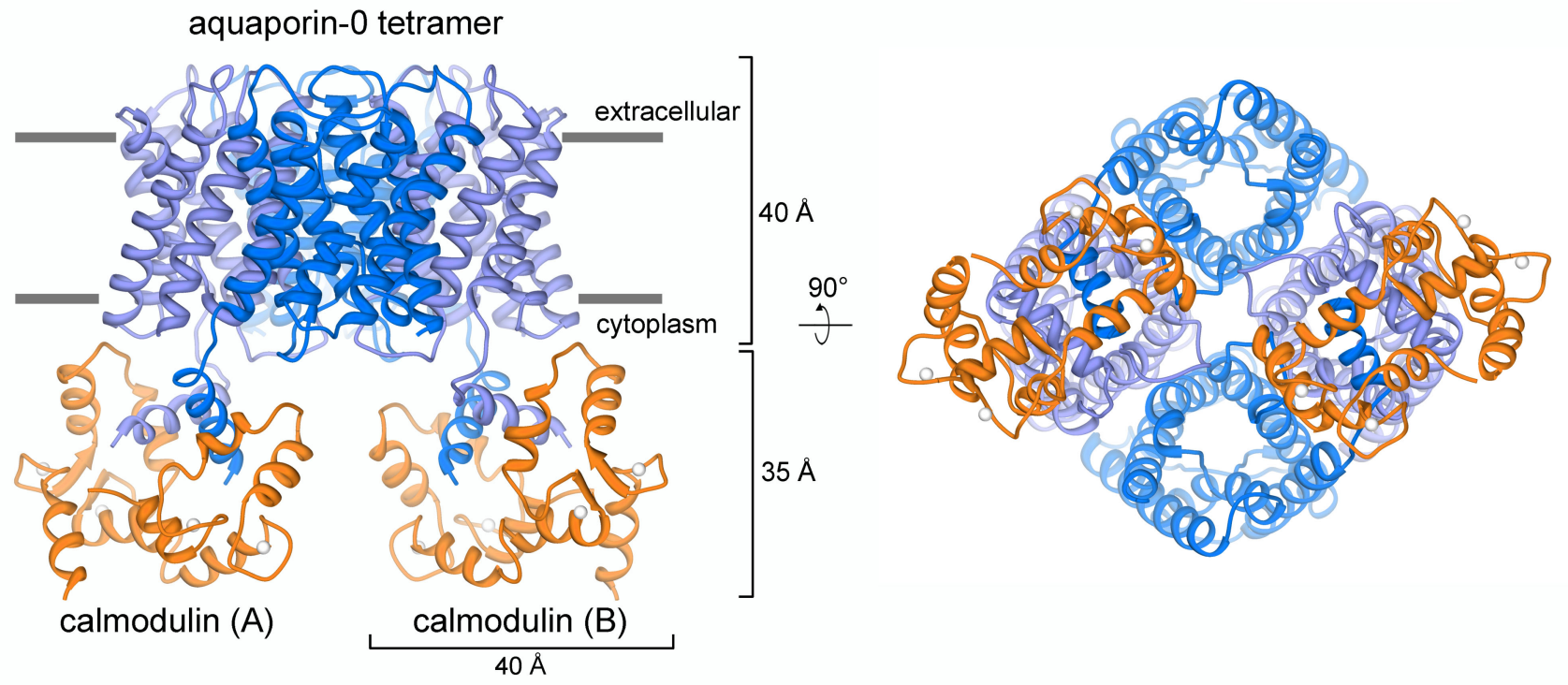

Figure 1. Pseudo atomic model of the aquaporin-0/calmodulin complex 